\title{
Testes de um Regenerador 2R e Conversor em comprimento de onda baseado em um único SOA no enlace de fibras KyaTera
}

\author{
Napoleão S. Ribeiro, Cristiano M. Gallep e Evandro Conforti
}

\begin{abstract}
Resumo - Um conversor em comprimento de onda baseado no efeito XGM utilizando apenas um amplificador óptico a semicondutor é apresentado. Este conversor apresenta boa capacidade de regeneração $(1<\Delta Q<4,5)$ para taxas de 0,6 a 13,5 Gbps. A caracterização foi realizada para sinais com modulação NRZ, incluindo testes no enlace de fibras do projeto KyaTera/FAPESP.
\end{abstract}

Palavras-chaves - Amplificador óptico a semicondutor, amplificadores ópticos, óptica não-linear, regeneração óptica.

Abstract - A XGM optical wavelength conversion scheme employing just one semiconductor optical amplifier with good regeneration capabilities $(1<\Delta Q<4.5)$ from 0.6 to $13.5 \mathrm{Gbps}$ is presented. The characterization was performed for NRZ streams, including tests on buried fiber of the KyaTera/FAPESP experimental testbed.

Keywords- Semiconductor optical amplifier, optical amplifiers, nonlinear optics, optical regeneration.

\section{INTRODUÇÃO}

Os novos serviços fornecidos pela internet têm aumentado a quantidade de informações, sendo necessária uma maior velocidade no tráfego de dados. Uma solução para maior rapidez é basear a novas redes em redes totalmente ópticas, nas quais o sinal será sempre utilizado no domínio óptico, sem conversões eletro-ópticas. Nestas redes, o amplificador óptico a semicondutor (SOA -semiconductor optical amplifier) se apresenta como um importante dispositivo para o processamento totalmente óptico do sinal [1]. Nas redes ópticas, o uso de conversores de comprimento de onda pode ser uma boa solução para se evitar congestionamento de dados bem como para ajudar no chaveamento de pacotes [2]. Além da conversão, em muitos casos, será necessária a regeneração do formato do bit no domínio totalmente óptico. A possibilidade de reformatação do pulso enquanto ocorre a conversão em comprimento de onda em redes totalmente

N. S. Ribeiro e E. Conforti trabalham no Departamento de Microondas e Óptica da Faculdade de Engenharia Elétrica e Computação, Unicamp.

C. M. Gallep trabalha na Divisão de Tecnologia de Telecomunicações, Centro Superior de Educações Tecnológica, Unicamp. E-mails: \{nribeiro,conforti\}@dmo.fee.unicamp.br, gallep@ceset.unicamp.br.

Este trabalho foi financiado em parte pela CAPES, CNPq e FAPESP, através dos projetos CEPOF e TIDIA-Kyatera, os quais o autor gostaria de agradecer. ópticas poderá ter grande utilidade em enlaces de média e grande distância operando em altas taxas.

Diversas técnicas para regeneração $2 \mathrm{R}$ (reamplificação e reformatação) baseadas em SOA têm sido propostas e testadas, como, por exemplo, usando: mistura de quatro ondas (FWM- four-wave mixing) [3], modulação cruzada de ganho (XGM -cross-gain modulation) [4], integração com interferômetro de Mach-Zehnder (MZI- Mach-Zehnder interferometer) [5], ou modulação cruzada de fase (XPM cross-phase modulation) [6]. A maioria dessas técnicas requer complexas implementações e/ou envolve pontos críticos de operação, mesmo nos casos mais simples baseados no efeito XGM [4].

O XGM é a técnica de conversão utilizando SOA mais simples de ser implementada. Na conversão em comprimento de onda via XGM, o sinal modulado em amplitude, de comprimento de onda $\lambda_{1}$, é injetado no amplificador e modula o ganho do SOA devido à sua saturação. Um segundo sinal, de onda contínua, em comprimento de onda $\lambda_{2}$, previamente escolhido, é também injetado no amplificador. Este sinal é então modulado pela variação do ganho do SOA. Dessa maneira, o sinal de saída em $\lambda_{2}$ terá a mesma informação que o sinal $\lambda_{1}$, embora com a fase invertida de $180^{\circ}$ [7].

Recentemente, apresentamos uma técnica bastante simples baseada no efeito XGM usando apenas um SOA, um laser, um isolador e um circulador. Esta simples configuração permite uma fácil e robusta operação [8].

Neste artigo, apresentamos resultados recentes de caracterizações de sinais ópticos modulados com um formato NRZ (Not Return to Zero), com obtenção de um melhor desempenho, operando com taxas de 4,25 a 11 Gbps. Nesta faixa de valores de taxa, foram obtidas melhorias de mais de 4 unidades no fator Q. Porém, mesmo para as piores condições, com taxas abaixo de 4,25 Gbps e acima de 11 Gbps, alguma regeneração pode ser obtida $(1<\Delta \mathrm{Q}<2,5)$.

\section{RESULTADOS EXPERIMENTAIS E DISCUSSÕES}

A regeneração é baseada principalmente, mas não somente, no comportamento em " $S$ " da eficiência do efeito XGM, que é comprimida para altas potências ópticas de entrada. Os fatores limitando o desempenho do 


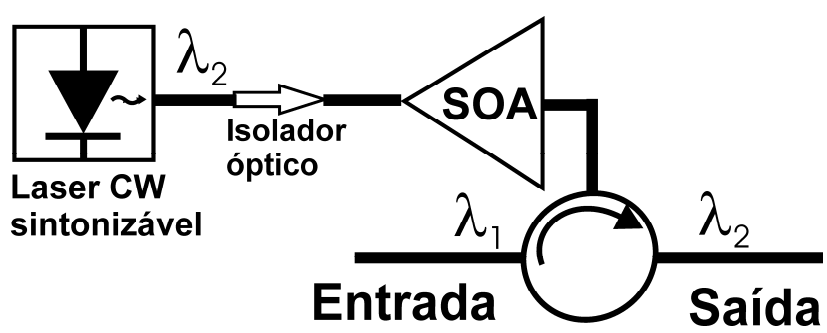

Fig.1. Esquema do conversor 2R totalmente óptico baseado em único SOA.

regenerador aqui apresentado são: a característica passa-baixa presente na amplificação do SOA que reduz a qualidade do diagrama de olho de saída para taxas maiores que $10 \mathrm{Gbps}$; e a dependência do padrão (pattern depedence) [9] do ganho óptico que é prejudicial para taxas menores que $3 \mathrm{Gbps}$. Porém, ao mesmo tempo em que limitam o desempenho, esses dois efeitos atuam de uma maneira positiva: a resposta passa-baixa pode minimizar flutuações (overshoots) que aparecem em baixas taxas de bits; a dependência do padrão também pode equalizar a resposta no tempo através da compressão do ruído em altas taxas de bit.

A montagem do conversor $2 \mathrm{R}$ totalmente óptico - laser sintonizável sem modulação (CW-Continuous Wave), SOA não-linear $(C I P)$ isolador e circulador óptico - está ilustrada na Fig. 1. A caracterização realizada foi semelhante à apresentada em [8]. O canal de entrada, uma portadora óptica modulada por um sinal pseudo-aleatório (NRZ) em $\lambda_{1}$, sintonizável ao longo da banda $\mathrm{C}$, é amplificado pelo SOA simultaneamente com um sinal CW em $\lambda_{2}$ (fixo em 1551,08 $\mathrm{nm}$ ). Os testes foram realizados, alterando-se: a qualidade do sinal de entrada, quantificada pelo fator Q; a taxa de bits $(0,6$

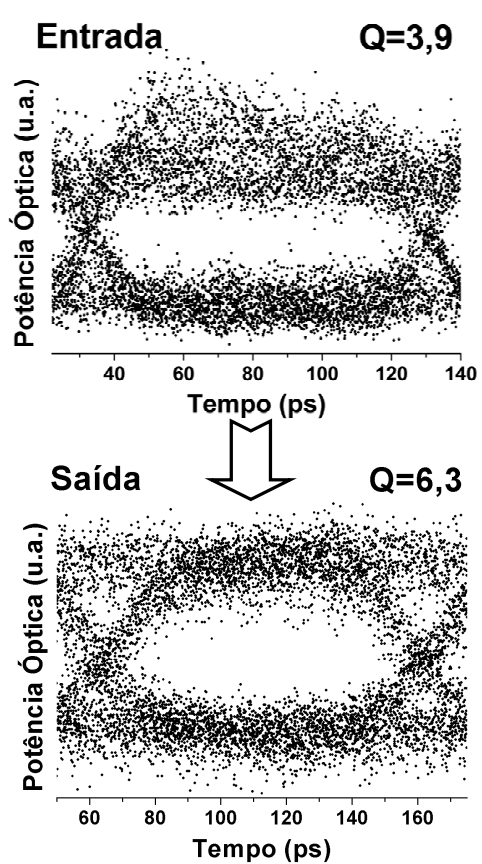

(a) até $13,5 \mathrm{Gbps})$; a potência óptica de entrada de ambos os canais, modulado e CW; e a distância espectral entre os dois canais (detuning), com $\Delta \lambda$ de 4 a $2 \mathrm{~nm}$. Os parâmetros dos sinais de saída - ganho óptico, diagrama de olho e fator Q foram medidos e os principais resultados estão apresentados neste trabalho.

Diagramas de olho para sinais de entrada e saída (conversão para maior comprimento de onda) e seus respectivos fatores Q para a taxa de 10,3125 Gbps estão apresentados na Fig. 2(a) e (b).

O pior caso do sinal de entrada $(\mathrm{Q}=3,9,(\mathrm{a}))$ foi obtido pelo uso de outro SOA como pré-amplificador, para compensar a perda de potência no modulador óptico. Esta préamplificação introduz efeitos de dependência do padrão, overshoots e ruído, tanto no nível alto ('1') quanto no nível baixo (' 0 ') de potência. $\mathrm{O}$ diagrama de olho com a qualidade média $(\mathrm{Q}=5,7,(\mathrm{~b}))$ ocorre para o caso em que a portadora óptica se propaga através de $18 \mathrm{~km}$ de fibras padrão monomodo que integram o enlace do projeto KyaTera [10]. A portadora óptica após os $18 \mathrm{~km}$ de fibra foi amplificada por um SOA e filtrado para a retirada do ruído ASE (Amplified Spontaneous Emission), apresentando distorção intensa no pulso devido à dispersão intrínseca. Em ambos os casos, ocorreu grande melhoria na abertura do diagrama de olho, com a redução dos overshoots e da variância dos níveis " 1 " e " 0 ". Estas melhorias são quantificadas pelo aumento do valor do fator $\mathrm{Q}$, respectivamente para $\mathrm{Q}=6,3$ e $\mathrm{Q}=10$. Para o caso da deterioração pela dispersão da fibra, os pulsos assumem uma forma triangular, e mesmo após a regeneração, o formato é mantido.
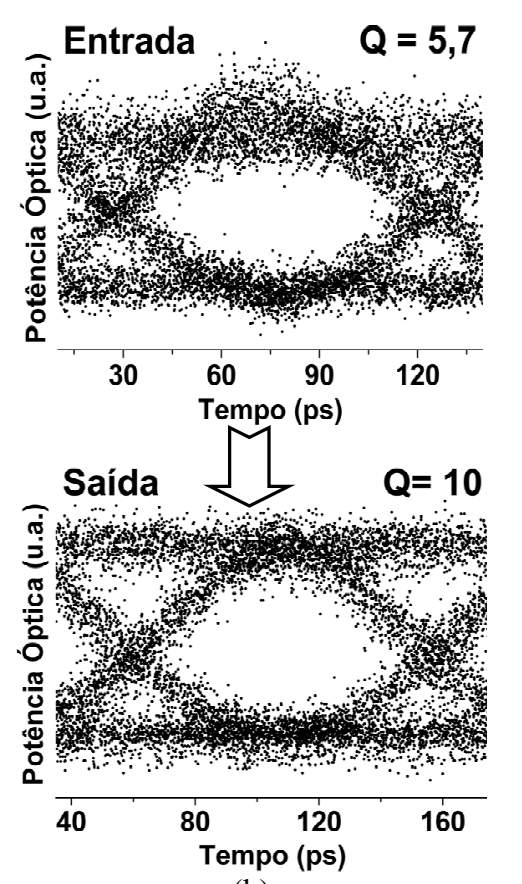

(b)

Fig.2. Diagramas de olho em 10,3125 Gbps para dois casos: (a) sinal de entrada com fator Q de 3,9 (deteriorado pela amplificação de um SOA) e saída convertida $(\Delta \lambda=1 \mathrm{~nm})$ e regenerada, com fator $\mathrm{Q}=6,3$; (b) sinal de entrada com fator $\mathrm{Q}$ de 5,7 (deteriorado pela dispersão das fibras do enlace do KyaTera) e saída convertida $(\Delta \lambda=1 \mathrm{~nm})$ e regenerada, com fator $\mathrm{Q}=10$. 


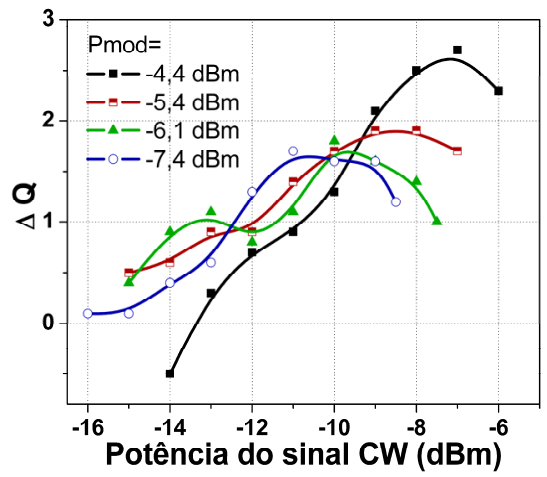

(a)

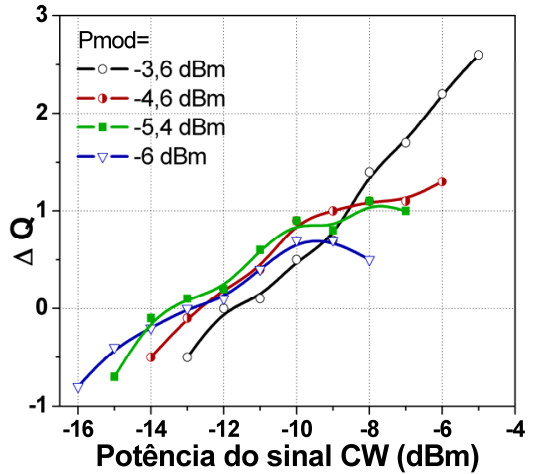

(b)

Fig.3. $\Delta$ Q versus potência do sinal de entrada (modulado e CW), para um canal de entrada deteriorado por: (a) dispersão da fibra (KyaTera), (b) outro SOA

As curvas apresentadas na Fig. 3 demonstram a dependência do desempenho do regenerador com a variação da potência óptica de entrada. O fator $\mathrm{Q}$ do sinal convertido pode ser maior que o do sinal original, quando a potência da portadora $\mathrm{CW}$ é menor que a potência do sinal modulado de 3 a $5 \mathrm{~dB}$. Isto acontece na maioria dos casos apresentados neste trabalho, ilustrando o comportamento em "S" da eficiência do XGM com relação à potência [4]. Além disso, esta relação entre as potências das portadoras $\mathrm{CW}$ e modulada é importante para se evitar a deterioração pelo efeito da dependência do padrão.

A distância espectral entre os sinais modulados e CW também foi variada. A portadora CW foi mantida em 1551,08 $\mathrm{nm}$ e a outra portadora variou de 1547,08 a $1553,08 \mathrm{~nm}$. O desempenho (melhora do fator Q) apresentou bons resultados para os dois casos de deterioração. Para o caso da entrada deteriorada pela dispersão da fibra foi obtida uma melhoria no $\Delta \mathrm{Q}$ superior a 3,5 , com resultado semelhante para conversão up (menores para maiores comprimentos de onda) e down (maiores para menos comprimentos de onda), mas com pior desempenho para conversão para o mesmo comprimento de onda. Para o caso do sinal de entrada deteriorado pelo amplificação de outro SOA, o mesmo comportamento foi observado, mas com menores valores de $\Delta \mathrm{Q}$, variando de 2,2 a 2,8 . Neste último caso, observamos melhores resultado para a conversão down.

$\mathrm{Na}$ Fig. 4., o estudo do desempenho do regenerador versus a taxa de bits está ilustrado para os dois casos de deterioração da entrada. Para este estudo, a potência óptica de entrada e o detuning $(1 \mathrm{~nm})$ foram fixados e dezessete padrões de taxa de bits de 622 Mbps a 13,5 Gbps foram usados. Na Fig. 4(a), pode-se observar que o $\Delta \mathrm{Q}$ aumenta de uma forma quase linear com a taxa de bits até 5 Gbps. Na faixa de 5 a 10,7 Gbps, observa-se os melhores valores para a melhoria do fator $\mathrm{Q}(\Delta \mathrm{Q}>4)$, com uma pequena queda no desempenho. Para as taxas mais altas, o desempenho decai rapidamente para o seu valor mínimo novamente. Na Fig. 4(b), o mesmo comportamento pode ser observado, mas com valores menores de $\Delta \mathrm{Q}$ (2,5 no máximo). Este comportamento corresponde ao esperado pelos efeitos descritos no início desta seção. Estes resultados demonstram que este regenerador pode ser muito bem aproveitado para sistemas que trabalhem com taxa até 10 Gbps. Entretanto, este regenerador está limitado a estas taxas, não pela configuração aqui apresentada, mas pelo tempo de vida dos portadores do SOA utilizado.

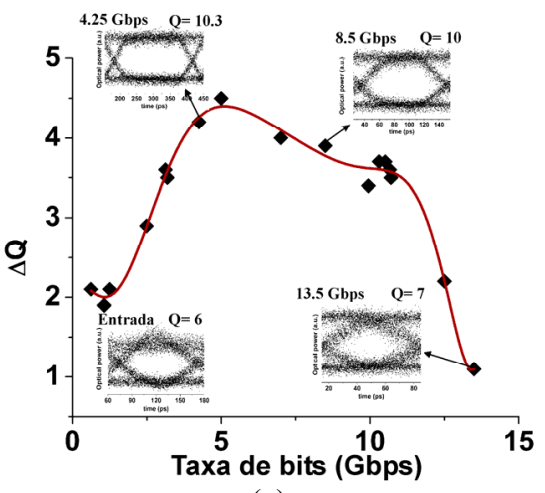

(a)

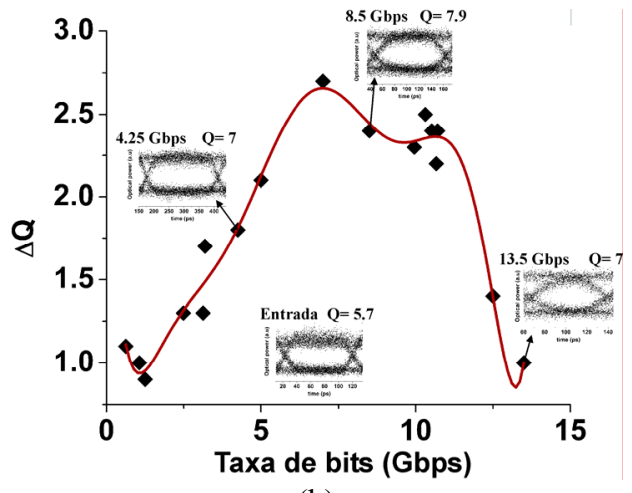

(b)

Fig.4. $\Delta$ Q versus taxa de bits, para o canal de entrada deteriorado por: (a) dispersão da fibra (KyaTera), (b) outro SOA. 


\section{CONCLUSÃO}

Bons resultados para regeneração $2 \mathrm{R}$ e conversão foram apresentados. O regenerador demonstrou melhor desempenho para os casos quando o sinal original foi deteriorado por um enlace de fibras. Foi observada regeneração tanto para conversões down como up, para diferentes detuning até 10 $\mathrm{nm}$ e para taxas de bits até $13,5 \mathrm{Gbps}$, com melhor desempenho para a faixa de 5 até 10,7 Gbps.

Observou-se que a regeneração ocorre para baixa potência óptica total de entrada $(<0,2 \mathrm{~mW})$ e que a operação é muito robusta e pouco sensível à polarização (devido ao SOA utilizado). Além da simplicidade e do bom desempenho, uma das principais vantagens desse conversor $2 \mathrm{R}$ é a alta velocidade de reconfiguração, limitada apenas pela sintonia do laser $\mathrm{CW}$, já que nenhum filtro sintonizável é necessário em um esquema contra-propagante. Uma outra vantagem é a de que este dispositivo como é baseado em SOA, pode ser facilmente integrado [11].

\section{REFERÊNCIAS}

[1] J.Leuthold, J. Jaques e S. Cabot, "All optical wavelength conversion and regeneration,"OFC 04, Los Angeles, CA, 2004, pg. 803.
[2] D. J. Blumental et al., "All-optical label swapping networks and technologies,"J. Lightwave Technol., vol. 18, pg. 2058-2075, Dez. 2000.

[3] H. Simos, A. Bogris e D. Syvridis, "Investigation of a $2 \mathrm{R}$ all-optical regenerator based on four-wave mixing in a semiconductor optical amplifier", IEEE J. Lightwave Techn., vol. 22, no.2, pg. 595-604 (2004).

[4] G. Contestabile, R. Proietti, N. Calabretta e E. Ciaramella, "Reshaping Capability of Cross-Gain Compression in Semiconductor Amplifiers", IEEE Photonics Techn. Letter, vol. 17, no.12, pg. 2523-2525, 2005.

[5] J. P. Wang. et. al, "Regeneration using an SOA-MZI in a 100-pass 10,000-km Recirculating Fiber Loop", CLEO/QELS 2007 - Conference on Lasers and Electro-Optics, paper CMZ1.

[6] H. Chayet, S. Ben Ezra, N. Shachar, S. Tzadok, S. Tsadka e J. Leuthold, "Regenerative all-optical wavelength converter based on semiconductor optical amplifier and sharp frequency response filter", Optical Fiber Communication Conference, OFC'04, Los Angeles , 2004.

[7]- T.Durhuus, B. Mikkelsen, C. Joergensen, S.L. Danielsen, K.E. Stubkjaer, "All-optical wavelength conversion by semiconductor optical amplifiers", Journal of Lightwave Tecnology, vol.14, no.6, pp 942$954,1996$.

[8] - N. S. Ribeiro, C.M. Gallep e E. Conforti;, "Wavelength Conversion and 2R-Regeneration Using One Semiconductor Optical Amplifier with Cross-Gain Modulation Compression", CLEO/QELS 2008 - Conference on Lasers and Electro-Optics, p. JWA28.

[9] J. Wang et al., "Patter Effect Removal Technique for Semiconductor Optical Amplifier based Wavelength Conversion," IEEE Photonics Techn. Letter, vol. 19, no.24, pg. 1955-1957 (2007).

[10] - www.kyatera.fapesp.br

[11] M. F. C. Stephens et. al, "All-optical regeneration and wavelength conversion in an integrated semiconductor optical amplifier/distributedfeedback laser," IEEE Photon. Technol. Lett., vol.11, pg. 979-981, 1999. 